\title{
Fold change rank ordering statistics: a new method for detecting differentially expressed genes
}

\author{
Doulaye Dembélé ${ }^{1,2^{*}}$ and Philippe Kastner ${ }^{1,3}$
}

\begin{abstract}
Background: Different methods have been proposed for analyzing differentially expressed (DE) genes in microarray data. Methods based on statistical tests that incorporate expression level variability are used more commonly than those based on fold change (FC). However, FC based results are more reproducible and biologically relevant.

Results: We propose a new method based on fold change rank ordering statistics (FCROS). We exploit the variation in calculated FC levels using combinatorial pairs of biological conditions in the datasets. A statistic is associated with the ranks of the FC values for each gene, and the resulting probability is used to identify the DE genes within an error level. The FCROS method is deterministic, requires a low computational runtime and also solves the problem of multiple tests which usually arises with microarray datasets.

Conclusion: We compared the performance of FCROS with those of other methods using synthetic and real microarray datasets. We found that FCROS is well suited for DE gene identification from noisy datasets when compared with existing FC based methods.
\end{abstract}

Keywords: Differentially expressed genes, Fold change, Averages of ranks, Microarray

\section{Background}

To select the differentially expressed (DE) genes in a microarray dataset with two biological conditions, the Fold Change (FC) which is calculated as a ratio of averages from control and test sample values was initially used [1,2]. Levels of change or cutoffs, (e.g. 0.5 for downand 2 for up-regulated) are used and genes under/above thresholds are selected. Then, other statistical methods were introduced. Many of these methods use three steps. First, a statistical test (e.g. Student's t-test or similar) is performed to obtain a p-value for each gene in the study. Second, these p-values are compared to a threshold which is chosen to have an acceptable False Discovery Rate (FDR), and a list of genes is obtained. Third, a selection is done from the above list using FC level thresholds for down- and up-regulated genes $[3,4]$. New

\footnotetext{
*Correspondence: doulaye@igbmc.fr

${ }^{1}$ Institut de Génétique et de Biologie Moléculaire et Cellulaire (IGBMC), INSERM U964, CNRS UMR 7104, Université de Strasbourg, 67404 Illkirch, France

2IGBMC Microarray and Sequencing Platform, 67404 IIIkirch, France

Full list of author information is available at the end of the article
}

statistical methods more adapted to microarray data were proposed [5-9]. The significance analysis of microarrays (SAM) method [6] provides an improvement to the ordinary Student $t$-test, as it imposes limits on the variability of genes, to exclude genes that do not change and which are associated with very low p-values. The performances of several methods were compared in [10-13] using two classes microarray datasets.

It has been shown that the FC based selection of genes leads to more reproducible results irrespective of the technology that is used [14-16]. Kadota et al. [12] proposed a FC based method, weighted average difference (WAD), which promotes highly expressed genes. WAD uses a weight factor for the average difference (AD) for each gene. The $\mathrm{AD}$ is obtained using log signals while the FC is computed from non-log signals. Comparative results in $[12,13]$ show that the WAD method is powerful for detecting DE genes in microarray data. However, like the simple FC based method, WAD does not associate an error to the list of selected genes. Hence, Farztdinov and McDyer [17] proposed a distributional fold change 
(DFC) test using the AD. A score is computed for each gene which is used for the ranking and selection of genes. The exact statistic of the DFC score is unknown even if it allows detection of weakly expressed genes that are lost with the WAD method. To take into account the variability in gene expression levels, many statistical methods were proposed. Some of these methods include the FC information to avoid the three step selection procedure mentioned above. McCarthy et al. [18] directly include a threshold for the gap between the averages in the Student t-test: $t$-test relative to a threshold (TREAT). The TREAT method is based on the linear model in [9]. In [19], the FC is combined with the hypothesis testing for assessing prediction error in the selection of the DE genes. More recently, Xiao et al. [20] combined the FC with a two samples statistical test p-value to obtain a score they called $\pi$-value. In all methods that can calculate a probability for each gene independently of the others, the problem of multiple tests arises. To avoid this problem, Qi et al. [21] used a mixture model. This model has four components corresponding to the expression status (yes or no) for the two biological conditions for each gene in the dataset. After model parameters estimation via an ExpectationMaximization type algorithm, the probabilities associated with genes are sorted, and a threshold is used to determine the list of genes given an error level. The method we propose here also avoids the problem of multiple tests and has a lower computational load.

Breitling et al. [22] devised a statistical method based only on the FC information. In their method, the FCs obtained in multiple pairs of control/test samples are ranked in decreasing order, and the product of the ranks (RP) for each gene is calculated. Combined probabilities $p^{\prime}$ are calculated by multiplying the RP values by a scalar factor which is determined using a permutation analysis to obtain an approximation of the expected RP values, see [22] for more details. A percentage of false-positive (PFP) is associated to each gene, and an acceptable PFP value is chosen to define the list of the DE genes. To select downregulated genes the sorting is done in increasing order and all subsequent steps are modified accordingly. The quality of the selection of the DE genes using the rank products method will depend mainly on the quality of the approximation of the expectation of the RP values using a permutation analysis. More recently, an exact statistic was proposed for the RP method [23]. However, for data with a large number of samples, the computational load is very heavy and this method is thus not recommended. Here, we propose another method based only on FC ranks. This method is very fast in comparison to the RP method, even for large numbers of samples in the dataset. In our method it is not necessary to search for up- and down-regulated genes in two separate steps. The statistic we obtain for each gene gives direct information on its status: down-regulated, up-regulated or not changed. This method also solves the problem of multiple tests which is usually encountered for microarray datasets. We exploit variations in the FC using several pairs of control/test samples. A statistics is associated to each gene, which results from the variation of the rank and the level of its FC.

\section{Methods}

\section{Preliminaries}

We consider a two conditions microarray experiment where $n$ probes (genes) are used with $m_{1}$ control and $m_{2}$ test samples. The number $n$ of probes is generally greater than 10,000 except for few species like yeast. Values for $m_{1}$ and $m_{2}$ are however small, most often lower than 100 . We note $\mathbf{x}_{i}=\left(\mathbf{x}_{i}^{c}, \mathbf{x}_{i}^{t}\right)=\left(x_{i 1}^{c} x_{i 2}^{c} \ldots x_{i m_{1}}^{c} x_{i 1}^{t} x_{i 2}^{t} \ldots x_{i m_{2}}^{t}\right)$ the values for the gene $i(i=1,2, \ldots, n)$ for the control samples $\left(x_{i j}^{c}, j=1,2, \ldots, m_{1}\right)$ and the test samples $\left(x_{i j}^{t}, j=1,2, \ldots, m_{2}\right)$, respectively. For a single color microarray, values $\left(x_{i j}^{c}, x_{i j}^{t}\right)$ are $\log _{2}$ levels, while they are $\log _{2}$ ratios for a two-color microarray. Here are examples of $\log _{2}$ transformed data for two genes (MACF1 and TREM2) taken from an experiment using Agilent microarrays (SurePrint, design 028004_D_F_20101102), with one color hybridization. Data for $M A C F 1$ are: $\mathbf{x}^{c}=$ $(11.1435,11.2860,11.2249,11.1258,11.0325,11.1108$, $11.3377,11.1821,11.0675,11.2381), \mathbf{x}^{t}=(11.0375,11.0792$, $10.9673,11.0367,11.1054,10.9261,11.0433,10.9484$, 10.9412, 10.8385); data for TREM2 are: $\mathbf{x}^{c}=(6.2856$, $6.4891,5.7799,6.1081,6.3129,6.3208,6.4826,6.2005$, 5.8922, 6.2148), $\mathbf{x}^{t}=(11.6792,8.1128,6.6253,6.8334$, $7.6417,7.5133,5.9633,7.4631,6.5666,7.6020)$. There are $m_{1}=10$ control and $m_{2}=10$ test samples. The FC and the Student t-test $\mathrm{p}$-value for MACF1 and TREM2 are $(0.8806,0.000248)$ and $(6.2570,0.01259)$, respectively. These results lead to the following two observations: a) a small Student $\mathrm{t}$-test $\mathrm{p}$-value is not necessary associated to a high FC, b) a high Student t-test p-value can be associated to a high FC. Indeed, the Student t-test statistic is calculated as $t=\frac{\overline{\mathbf{x}}^{t}-\overline{\mathbf{x}}^{c}}{s_{p}}$, where $\overline{\mathbf{x}}^{t}$ and $\overline{\mathbf{x}}^{c}$ are average levels of the control and test samples respectively, $s_{p}^{2}$ is the combined variance from those of the control and test samples: $s_{p}^{2}=\frac{\left(m_{1}-1\right) s_{1}^{2}+\left(m_{2}-1\right) s_{2}^{2}}{m_{1}+m_{2}-2},\left(s_{1}^{2}\right.$ and $s_{2}^{2}$ are variances of $\mathbf{x}^{t}$ and $\left.\mathbf{x}^{c}\right)$. For the same average difference $\left(\overline{\mathbf{x}}^{t}-\overline{\mathbf{x}}^{c}\right)$, a small $s_{p}^{2}$ can lead to high $t$ (small $\mathrm{p}$-value), on the other hand, a large $x_{p}^{2}$ can lead to a small $\mathrm{t}$ (high p-value). Hence, a small (high) average difference can have a small (high) Student t-test $\mathrm{p}$-value. The variances of data for genes MACF1 and TREM2 given above are 0.008 and 1.26, leading to t-statitics equal to 4.549 and 2.711 respectively. These observations are highlighted by Xiao et al. [20] and correspond to the SFSV (small fold change, small variance) and the LFLV (large fold change, large variance), respectively. 
For the proposed method, the probability of the statistic obtained is close to zero (one) for down-(up)regulated genes. Using the method described below, the probabilities associated to the statistics obtained for MACF1 and TREM2 are 0.12105 and 0.9964 , respectively. These values mean that MACF1 does not change and that TREM2 is up-regulated.

\section{Description of the method}

Being given expression values for $n$ genes in $m_{1}$ control and $m_{2}$ test samples, we perform $k \leq m_{1} m_{2}$ pairwise comparisons and compute FCs for each gene (test/control). In each comparison, the $n$ FCs obtained are sorted in increasing order and their corresponding ranks are associated to genes. Hence, for gene $i$, we get a vector $\mathbf{r}_{i}=\left(r_{i 1} r_{i 2} \ldots r_{i j} \ldots, r_{i k}\right)$ where $r_{i j}$ corresponds to the rank of the FC for gene $i$ in the $j$ comparison $(j=1, \ldots, k)$. The ranks are integers that belong to the set $\{1,2, \ldots, n\}$. To deal with ties, the rank values are adjusted in such a way that their sum reaches the same total as that reached if there is no tie. By construction, knowledge of one component of the vector $\mathbf{r}_{i}$ does not allow to predict the another ones. This leads to an independence of the ranks associated to pairwise comparisons. Hence, the components of the vector $\mathbf{r}_{i}$ can be considered as samples of the true unknown rank associated to gene $i$. Ideally, the same rank should be assigned to each gene in the $k$ comparisons. The probability of this event is $\frac{1}{n^{k+1}}$ and is unlikely to happen. Hence, the averages of ranks (a.o.r) $\bar{r}_{i}, i=1,2, \ldots, n$, will vary between a minimum $a=\min _{i}\left\{\bar{r}_{i}\right\}$ and a maximum $b=\max _{i}\left\{\bar{r}_{i}\right\} . \bar{r}_{i}$ is an average of components in $\mathbf{r}_{i}$. We can order all the a.o.r $\bar{r}_{i}$ from the minimum to the maximum and write: $\overline{\mathbf{r}}=\left[a,\left(a+\delta_{1}\right),\left(a+\delta_{1}+\delta_{2}\right), \ldots,(a+\right.$ $\left.\left.\delta_{1}+\ldots+\delta_{n-2}\right), b\right]$ where scalars $\delta_{i}(i=1, \ldots, n-1)$ are the differences between consecutive ordered a.o.r, and $\overline{\mathbf{r}}$ is a vector with all $\bar{r}_{i}$. Without loss of generality, let us assume that the differences $\delta_{i}$ have the same value which is approximated by their mean: $\delta=\frac{b-a}{n-1}$. Hence, the ordered a.o.r $\bar{r}_{i}, i=1,2, \ldots, n$, can then be writen as: $\overline{\mathbf{r}}=[a,(a+\delta),(a+2 \delta),(a+3 \delta), \ldots,(a+(n-1) \delta)]$. Our method is based on the behavior of the ordered a.o.r $\overline{\mathbf{r}}$ and we have the following theorem.

Theorem 1. When the number $k$ of the pairwise comparisons grows, the ordered averages of ranks (a.o.r) $\overline{\mathbf{r}}$ have a normal distribution. The mean of this distribution is $\frac{a+b}{2}$ and its variance is $\frac{n^{2}-1}{12} \delta^{2}$, where $a$ and $b$ are the minimum and the maximum of the observed a.o. $r \overline{\mathbf{r}}$, respectively. $\delta$ is an average difference between consecutive ordered a.o.r. $\overline{\mathbf{r}}$.

Proof. We note $\bar{r}_{i}=\frac{1}{k} \sum_{j=1}^{k} r_{i j}$ the average of the components in $\mathbf{r}_{i}$. Let us note the expectation and the variance of the ranks in vector $\mathbf{r}_{i}$ by $E\left\{\mathbf{r}_{i}\right\}=R_{i}$ and $\operatorname{Var}\left\{\mathbf{r}_{i}\right\}=\sigma_{R_{i}}^{2}$.
Using the central limit theorem ([24], page 259) it follows that the quantity $\frac{\sqrt{k}}{\sigma_{R_{i}}}\left(\bar{r}_{i}-R_{i}\right)$ converges to a normal distributed variable having a mean of zero and a variance of one when $k$ is high. Hence, we obtain $n$ normal distributed variables $R_{i}$.

From the selection theorem ([24], page 267), the sequence of the $n$ normal variables has a normal distribution. Expectation and variance of variable $R_{i}$ are given by

$$
\begin{aligned}
E\left\{R_{i}\right\} & =\frac{1}{n} \sum_{i=1}^{n} R_{i} \\
\operatorname{Var}\left\{R_{i}\right\} & =E\left\{R_{i}^{2}\right\}-\left(E\left\{R_{i}\right\}\right)^{2}
\end{aligned}
$$

By replacing each normal variable $R_{i}$ by the average of samples from which it is derived and by using $\sum_{i=1}^{n} i=$ $\frac{n(n+1)}{2}$, we have

$$
\begin{aligned}
E\left\{R_{i}\right\} & =\frac{1}{n}(a+(a+\delta)+(a+2 \delta)+\ldots+(a+(n-1) \delta)) \\
& =\frac{1}{n}(n a+(1+2+\ldots+(n-1)) \delta) \\
& =\frac{1}{n}\left(n a+\frac{n(n-1)}{2} \delta\right)=a+\frac{(n-1)}{2} \delta=\frac{a+b}{2}
\end{aligned}
$$

By using $\sum_{i=1}^{n} i^{2}=\frac{n(n+1)(2 n+1)}{6}$, we also have

$$
\begin{aligned}
E\left\{R_{i}^{2}\right\}= & \frac{1}{n}\left(a^{2}+(a+\delta)^{2}+(a+2 \delta)^{2}\right. \\
& \left.+\ldots+(a+(n-1) \delta)^{2}\right) \\
= & \frac{1}{n}\left(n a^{2}+2(1+2+\ldots+(n-1)) a \delta+\left(1^{2}+2^{2}\right.\right. \\
& \left.\left.+\ldots+(n-1)^{2}\right) \delta^{2}\right) \\
= & \frac{1}{n}\left(n a^{2}+n(n-1) a \delta+\frac{n(n-1)(2 n-1)}{6} \delta^{2}\right) \\
= & a^{2}+(n-1) a \delta+\frac{(n-1)(2 n-1)}{6} \delta^{2}
\end{aligned}
$$

Using equations 9 and 5, relation 2 leads to

$$
\begin{aligned}
\operatorname{Var}\left\{R_{i}\right\}= & a^{2}+(n-1) a \delta+\frac{(n-1)(2 n-1)}{6} \delta^{2} \\
& -\left(a+\frac{(n-1)}{2} \delta\right)^{2} \\
= & (n-1)\left(\frac{2 n-1}{6}-\frac{n-1}{4}\right) \delta^{2}=\frac{n^{2}-1}{12} \delta^{2}
\end{aligned}
$$


Theorem 1 is the basis of our method for selecting the DE genes. The presence of outlier sample(s) will impact the a.o.r. $\bar{r}_{i}$ value associated to gene $i$. Thus, we use a trimmed mean by removing a percentage of low and large ranks in the calculation of $\bar{r}_{i}$. This percentage is a tuning parameter of the FCROS method which is summarized as follows (see also Figure 1).

\section{FCROS algorithm}

1. Given microarray data having $m_{1}$ control and $m_{2}$ test samples, perform $k \leq m_{1} m_{2}$ pairwise comparisons and compute FCs for genes (test/control). These FCs are sorted in increasing order and their corresponding ranks are associated to genes.

2. Compute a robust average of rank $\bar{r}_{i}$ for each gene $(i=1,2, \ldots, n)$ using its $k$ values. This can be done using a trimmed mean. Sort values of $\overline{\mathbf{r}}$ by increasing order to get $\overline{\mathbf{r}}^{s}$ where $\bar{r}_{1}^{s} \leq \bar{r}_{2}^{s} \leq \ldots \leq \bar{r}_{n}^{s}$.

3. Compute sample mean $\bar{R}=\frac{1}{n} \sum_{i=1}^{n} \bar{r}_{i}$ and sample variance $\hat{\sigma}_{R}^{2}=\frac{1}{n-1} \sum_{i=1}^{n}\left(\bar{r}_{i}-\bar{R}\right)^{2}$. The minimum average rank is $a=\bar{r}_{1}^{s}$, and the maximum average rank is $b=\bar{r}_{n}^{s}$. Compute differences between consecutive terms of $\bar{r}_{i}^{s}$ and then derive an estimate for parameter $\delta$ as the mean of the obtained differences: $\hat{\delta}=\frac{1}{n-1} \sum_{i=1}^{n-1}\left(\bar{r}_{i+1}^{s}-\bar{r}_{i}^{s}\right)$.

4. Use $\bar{R}$ and $\hat{\sigma}_{R}^{2}$ as parameters of a normal distribution and associate probabilities to genes through their $\bar{r}_{i}$ values. Since a p-value refers to the probability associated with a hypothesis testing statistic, we call probabilities associated to fold change ranks ordering statistics f-values. A f-value close to 0.5 corresponds to an equally expressed (EE) gene, while down- and up-regulated genes have f-values close to 0 and 1 , respectively.

5. Set error levels, $\alpha_{1}$ and $\alpha_{2}$, for down- and up-regulated genes to select the DE genes.

We use standardized ranks, i.e. each component in $\mathbf{r}_{i}$ is divided by $n$. Hence, the mean and standard deviation in step 3 of the algorihm above should be divided by $n$. In the FCROS algorithm, necessary parameters are computed from the dataset except the trimmed mean percentage parameter noted trim. Theorem 1 gives theoretical values for many parameters, more precisely $\delta=\frac{b-a}{n-1}, \bar{R}=\frac{b+a}{2 n}$ and $\sigma_{R}^{2}=\left(\frac{1}{12}-\frac{1}{12 n^{2}}\right) \delta^{2}$. For the ideal situation $(a=\delta=1$, $b=n)$ theoretical mean and variance are $\frac{1}{2}+\frac{1}{2 n} \approx \frac{1}{2}$ and $\frac{1}{12}-\frac{1}{12 n^{2}} \approx \frac{1}{12}$, respectively. Let us examine the role of parameters $k, \delta$ and trim.

Parameter $\boldsymbol{k}$ The size of the integer $k$ allows to fulfill the conditions to apply the central limit theorem, higher values for $k$ being optimal. The maximum value $m_{1} m_{2}$ for $k$ is determined by the number of control and test samples in the dataset.

Parameter $\delta$ Parameter $\delta$ takes its value in the interval $[0,1]$. The ideal value $\delta=1$ is unlikely to be obtained. A small value of the parameter $\delta$ leads to a small variance $\hat{\sigma}_{R_{i}}^{2}$. This will happen when the difference between upper and lower bounds of the ordered a.o.r $\bar{r}_{i}$ becomes smaller, i.e., if the observed changes in the ranks associated with genes are large, so that the a.o.r will tend to move away from the ideal bounds 1 and $n$. We can consider the parameter $\delta$ as a fraction of the dataset size range: $\delta=\frac{b-a}{n-1}=\frac{n}{n-1}(\beta-\alpha)$ where $\beta=b / n$ and $\alpha=a / n$. From this point of view, a value of $\delta$ equal to 0.98 can correspond to $(b=0.99 n, a=$ $0.01 n)$ and is better than a value for $\delta$ equal to 0.66 which can correspond to the bounds $\left(b=\frac{5}{6} n, a=\frac{1}{6} n\right)$ which are more distant from $n$ and 1 . We provide numerical values for $\delta$ in Additional file 1: Figures S3 and S5 using synthetic and real microarray datasets.

Parameter trim To have a robust estimation of the o.a.r $\bar{r}_{i}$ we use a fraction of ranks associated to gene $i$. Parameter trim allows to delete some ranks from each end (small and high ranks) before computing the mean. Thus, a value for trim equal to 0.1 means that $80 \%$ of the ranks for gene $i$ are used to calculate $\bar{r}_{i}$.

\section{Results and discussion}

To evaluate the performance of the FCROS method, we used synthetic and real microarray datasets. We compared the results obtained with our method to those obtained using six other methods: the simple fold change (FC), the weighted average difference (WAD), the rank product (RP), the Student t-test (Ttest), the significant analysis
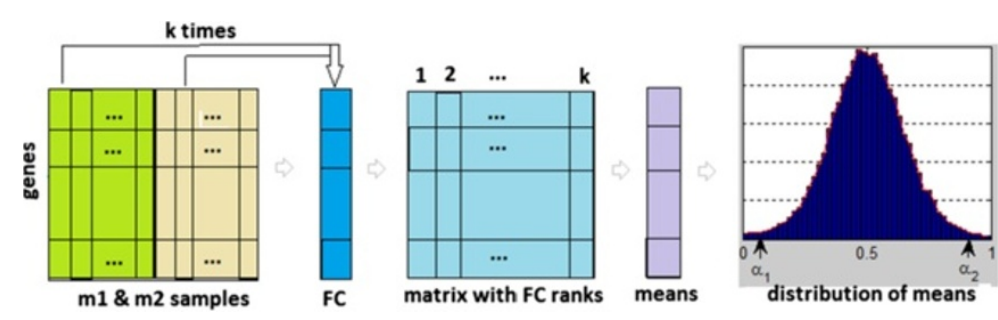

Figure 1 Steps of the FCROS method. Scheme depicting the steps of the FCROS method. 
of microarray (SAM) and the t-test relative to a threshold (TREAT) methods. All calculations were performed on the same computer (Personnal Computer equiped with i7-2640M processor, 8GB of RAM, under Microsoft Windows Professional 7) and $R$ version 3.0.1. We implemented a new $R$ package, fcros, which is available from the comprehensive $\mathrm{R}$ archive network (http://cran.r-project. org/web/packages/fcros/) [25]. For all results presented, the trim parameter was set to 0.3. We also used three other R packages, samr [26], RankProd [27] and limma [9] with their default settings, but the parameter huge in the RankProd package was set to TRUE. We used the ROC (receiver operating characteristics) $\mathrm{R}$ package [28] to obtain an area under a ROC curve (AUC) for methods when true DE genes are available. For real microarray datasets, no prefiltering was performed before searching for the DE genes except for one dataset.

\section{Synthetic datasets}

We used the microarray data simulation model (MADSIM) described in [29] to generate synthetic data with known characteristics; in particular, the indexes of the DE genes are known. A R package implementing MADSIM is available from the comprehensive $\mathrm{R}$ archive network [30].

Synthetic datasets 1 To evaluate the behavior of the FCROS method in the presence of noise, we used three different values for the parameter $\sigma_{n}$ of MADSIM. 100 simulations corresponding to different initializations ( $r$ seed $=10,20,30, \ldots, 1000$.) were used. All other parameters of MADSIM were set to their default settings. More precisely, $m_{1}=m_{2}=7, n=10,000$ and the proportion of DE genes was set to 0.02 . These settings lead to an expected number of $200 \mathrm{DE}$ genes. Additional file 1: Figure S1 shows the M-A plot [31] for 3 datasets which correspond to 3 setting values for parameter $\sigma_{n}$ of MADSIM.

For each dataset, i.e. corresponding to a given value for parameters $r$ seed and $\sigma_{n}$, we used the FCROS and the six other methods to determine the DE genes, the number of which was set to that of true DE genes. The genes selected by each method were split into two sets: true and false DE genes. The results are plotted in Figure 2, which shows that the FCROS, FC, RP, SAM and TREAT methods performed well, and that the Ttest and WAD methods had a lower performance. Of note, in these tests, the runtime of the FCROS method was more than hundred times faster than that of the RP method.

Synthetic dataset 2 We used MADSIM to generate a dataset with $m_{1}=m_{2}=15$ and default settings for all other parameters. This dataset has 198 true DE genes. Additional file 1: Figure S2 shows the M-A plot [31] of this synthetic dataset. Synthetic dataset 2 was used in different scenarios where we specified different values $\left(m_{1} x m_{2}\right)$ for the control and test samples, and performed the following steps: a) random selection of $m_{1}$ control and $m_{2}$ test samples from their respective sets, b) running the FCROS and the six other methods, c) selection of the top $198 \mathrm{DE}$ genes for each method, and assignment of a value of 1 to true DE genes and of 0 to all other genes. These 3 steps are repeated 100 times and the total occurences of 1 for each method and $m_{1} x m_{2}$ combination is calculated as its score, which is thus comprised between 0 and 100 .

Results obtained for the seven methods are shown in Figure 3. The FCROS, FC, RP and TREAT methods had similar power of detection, with the TREAT and RP methods exhibited a slightly better performance for the case $3 \times 3$. The Student t-test and the WAD methods gave worse results than the other methods. In addition, we performed another run using the complete dataset $\left(m_{1}=15\right.$, $\left.m_{2}=15\right)$. Table 1 shows the results obtained as well as the AUC (area under a ROC curve) values for the seven methods. The TREAT, FC and RP methods had the lowest error while the Ttest and WAD methods had the highest one.

We recorded values for parameter $\delta$ for runs of the FCROS method. Results obtained are plotted in Additional file 1: Figure S3. The values for $\delta$ are close to 1 if control and test samples are not randomized, see panel A of Additional file 1: Figure S3. These values decrease towards 0.7 for random and an increasing number of control and test samples, see panel B of Additional file 1: Figure S3.

\section{Microarray data}

We used seven microarray datasets to evaluate the performance of the FCROS method. All data were generated with the Affymetrix technology. The first dataset ("Platinum Spike") is from [32] and consists of 18 spike-in samples ( 9 controls versus 9 tests). This dataset is available from the Gene Expression Omnibus website under the accession number GSE21344. The next six post-processed datasets are available from [33]. For the second dataset, 58 diffuse large B-cell lymphoma (DLBCL) patients and 19 follicular lymphoma patients were used [34]. The third dataset (Prostate) consists of 102 samples using 50 nontumor and 52 tumor prostate patients [35]. The fourth dataset (Colon) consists of 22 control and 40 colon cancer samples [36]. For the fith dataset (Leukemia), 47 acute lymphoblastic leukemia and 25 acute myeloblastic leukemia patients were used [5]. The sixth dataset (Myeloma) was obtained using 36 patients without and 137 patients with bone lytic lesions [37]. For the seventh dataset (ALL-1), 128 different individuals (95 B-cell leukemia and $33 \mathrm{~T}$-cell leukemia) were used [38].

"Platinum Spike" dataset We downloaded the Affymetrix CEL format files from the GEO website 

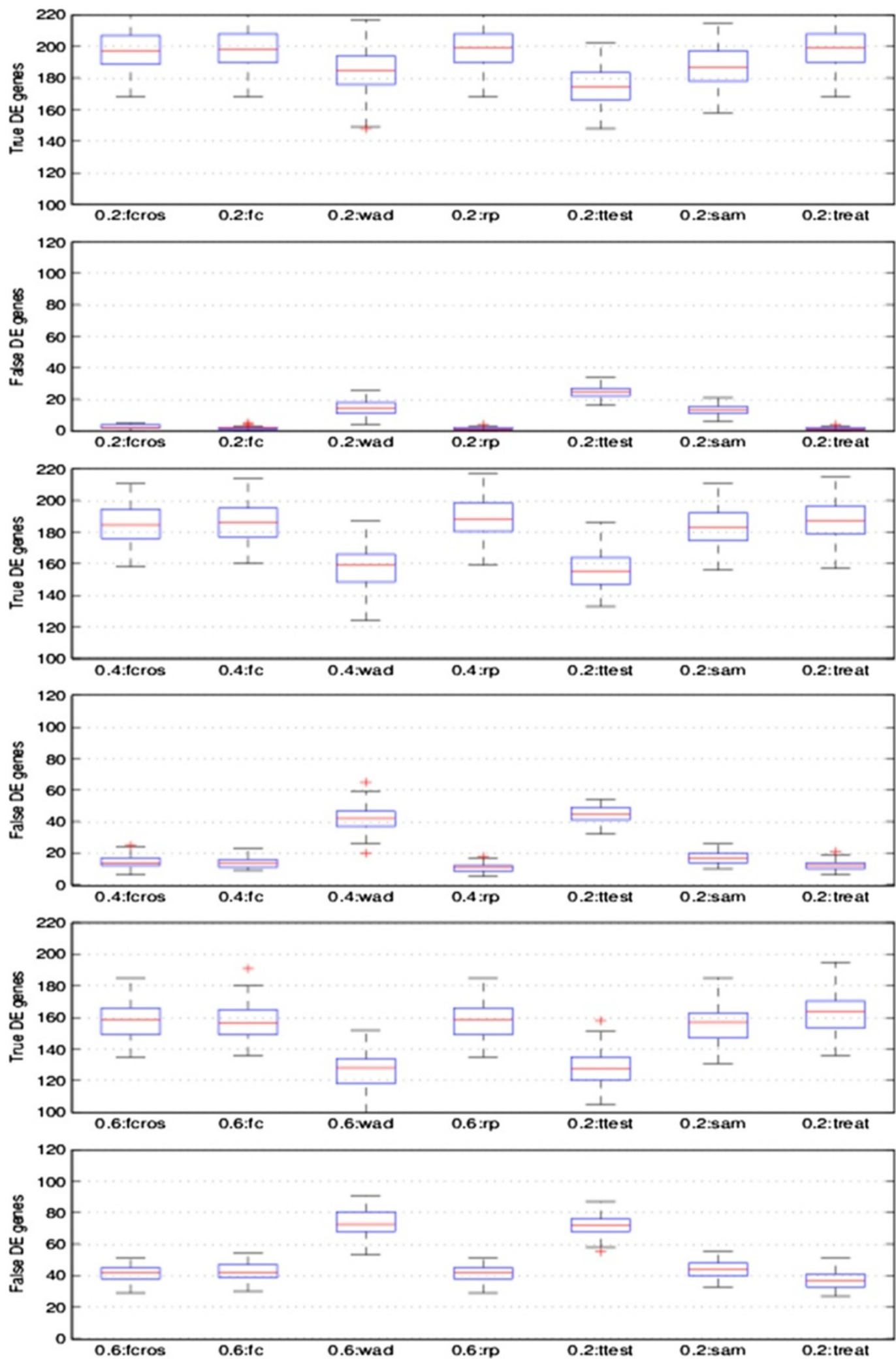

Figure $\mathbf{2}$ Comparison of the performance of methods with synthetic data associated with varying levels of noise. Boxplots of the number of the true and false DE genes using the FCROS (fcros), FC (fc), WAD (wad), RP (rp), Student t-test (ttest), SAM (sam) and TREAT (treat) methods. The noise level parameter $\sigma_{n}$ was set to $0.2,0.4$ and 0.6 .100 simulations were used for these results. 

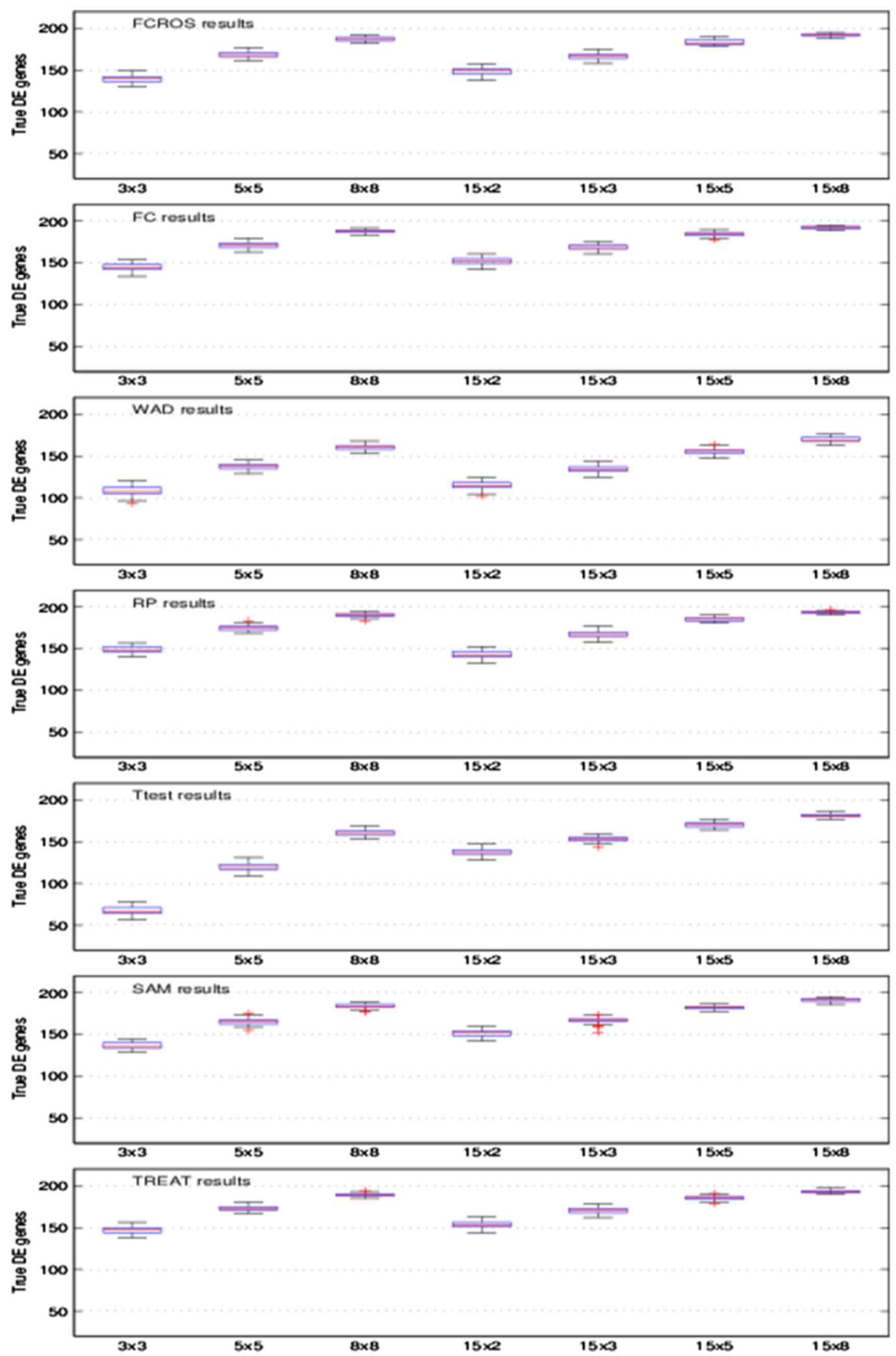

Figure 3 Comparison of the performance of methods with synthetic data and varying sizes of sample groups. Number of the true $D E$ genes using the FCROS, FC, WAD, RP, Ttest, SAM and TREAT methods. 
Table 1 Comparative results for the synthetic dataset 2

\begin{tabular}{llllll}
\hline Method & Thresholds & Selection & False & Error & AUC \\
\hline FCROS & $0.002348226,0.998$ & 198 & 2 & $1.0 \%$ & 0.9999928 \\
FC & 1.5922 & 198 & 1 & $0.5 \%$ & 0.9999933 \\
WAD & 1.2072 & 198 & 17 & $8.5 \%$ & 0.9981039 \\
RP & 0.00009 & 198 & 1 & $0.5 \%$ & 0.9999985 \\
Ttest & 0.00059892 & 198 & 6 & $3 \%$ & 0.9999356 \\
SAM & 0.0007 & 198 & 3 & $1.5 \%$ & 0.9999691 \\
TREAT & 0.00078531 & 198 & 1 & $0.5 \%$ & 0.9999990 \\
\hline
\end{tabular}

Synthetic dataset 2: Selection, errrors and AUC for the seven methods. Error is calculated as the number of false genes detected divided by 198 , the number of true DE genes in the dataset.

(GSE21344) and used the RMA (robust multi-array average) method to obtain signals for probes [39]. We downloaded the designated FC associated to probes from: www.biomedcentral.com/content/supplementary/ 1471-2105-11-285-s5.txt (accessed on 23 september 2013). Using this file, we retained 18952 probes, among which 1940 are known as DE. Each of these probes has an observed FC obtained using RMA normalized data and a designated FC read from the file we downloaded. Additional file 1: Figure S4 shows the M-A plot [31] of the "Platinum Spike" dataset.

We ran the seven methods and selected the top 1940 probes which were then crossed with the set of the designated DE probes. The results are summarized in
Table 2. In this Table, the "Status" indicates whether the gene is equally expressed (EE) or differentially expressed (DE). The "A vs B" is the designated fold change and "Number" is the number of probes for a status. The AUC values and the percentages of false detection for the seven methods are also given. The WAD, Ttest, SAM and TREAT methods were more efficient for $\mathrm{A}$ vs $\mathrm{B}=0.83$ than the other methods (FCROS, FC and RP). For all other $A$ vs $B$ cases, the seven methods have a similar detection efficiency. The results obtained with the FCROS, RP, SAM and TREAT methods are represented in a Venn diagram in Figure 4A. This figure shows that the large majority of DE genes were similarly detected by all 4 methods. The $t-$ test based methods (SAM and TREAT) were slightly more sensitive than the FC-based methods as they detected 39 DE genes (mostly with low FC values, Figure 4C) that neither the FCROS or RP methods detected.

We further compared the performances of the $4 \mathrm{FC}$ based methods, Figure 4B. This comparison revealed that the WAD method outperformed the other methods, as it specifically detected $71 \mathrm{DE}$ genes (Figure 4C). However, it should be noted that the FC, FCROS and RP methods collectively identified $68 \mathrm{DE}$ genes (mostly with low expression values, Figure 4D) that were not detected by the WAD method.

Results for six sets of microarray data We used the DLBCL, Prostate, Colon, Leukemia, Myeloma and ALL-1

Table 2 Comparative results for the Platinum spike dataset

\begin{tabular}{llllllllll}
\hline Status & A vs B & Number & FCROS & FC & WAD & RP & Ttest & SAM & TREAT \\
\hline EE & 0 & 13337 & 216 & 220 & 19 & 221 & 142 & 203 & 176 \\
DE & 0.25 & 192 & 161 & 161 & 158 & 161 & 162 & 161 & 161 \\
DE & 0.28 & 174 & 162 & 163 & 154 & 163 & 157 & 163 & 163 \\
DE & 0.4 & 163 & 132 & 134 & 127 & 133 & 131 & 133 & 133 \\
DE & 0.66 & 189 & 151 & 155 & 147 & 154 & 89 & 149 & 139 \\
DE & 0.83 & 166 & 46 & 43 & 111 & 40 & 118 & 60 & 83 \\
EE & 1 & 3426 & 52 & 49 & 232 & 50 & 158 & 56 & 77 \\
DE & 1.5 & 167 & 134 & 134 & 131 & 135 & 114 & 135 \\
DE & 1.7 & 166 & 150 & 150 & 141 & 150 & 145 & 149 & 131 \\
DE & 2 & 184 & 161 & 161 & 158 & 162 & 162 & 162 \\
DE & 3 & 98 & 94 & 94 & 92 & 94 & 94 & 94 \\
DE & 3.5 & 445 & 397 & 394 & 382 & 396 & 388 & 393 \\
EE/DE & MC & 231 & 74 & 74 & 77 & 73 & 71 & 74 \\
EE/DE & MF & 14 & 10 & 8 & 11 & 8 & 9 & 39 \\
\hline
\end{tabular}

Platinum Spike dataset: number of genes detected by each method for a given change condition. EE and DE are for equally expressed and differentially expressed, respectively. "A vs $B$ " is the designated fold change and "Number" is the number of probes for each change condition. Detection error and the AUC are also given at the bottom of the Table. 


\section{A}

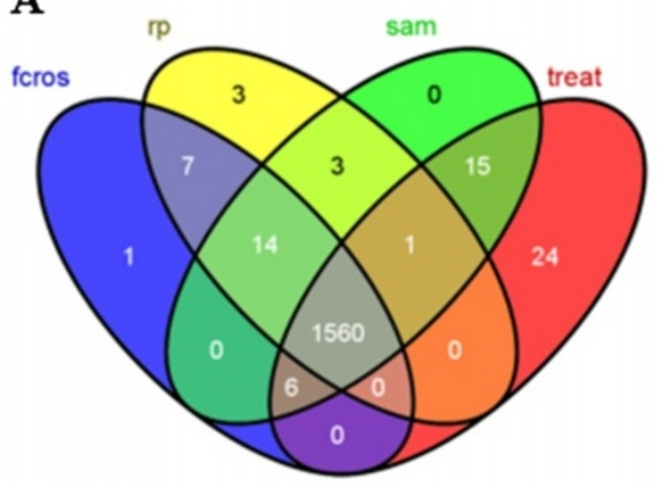

B

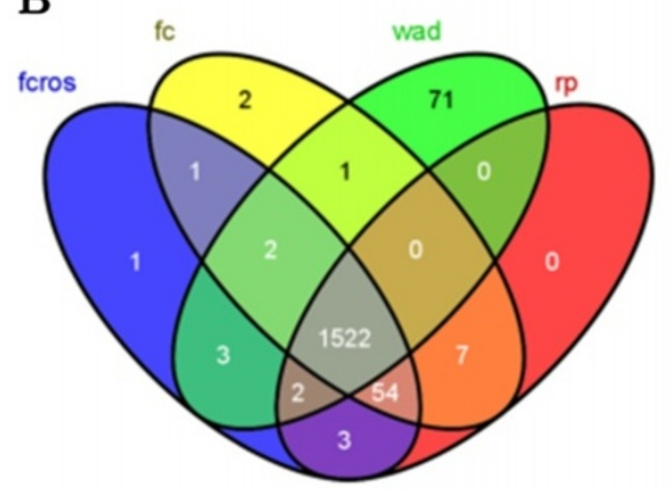

C

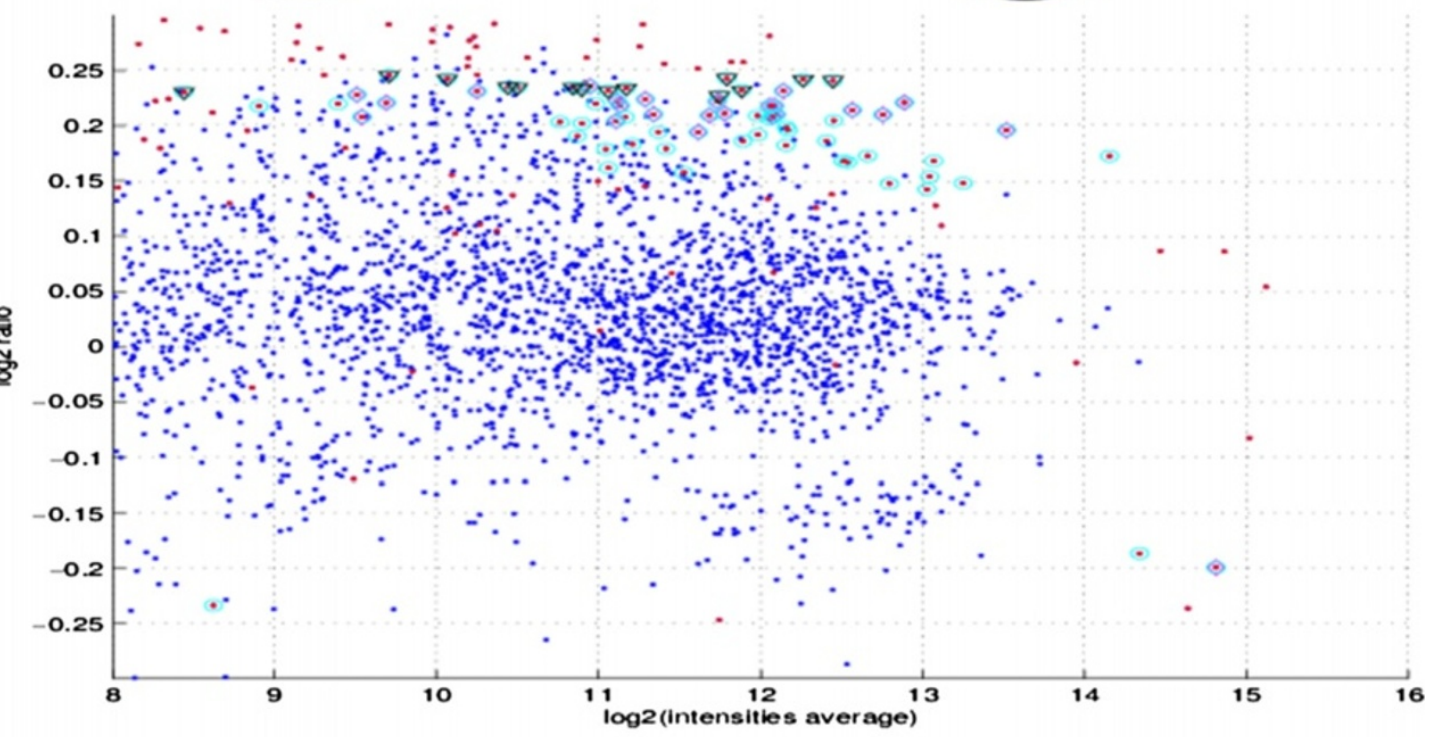

D

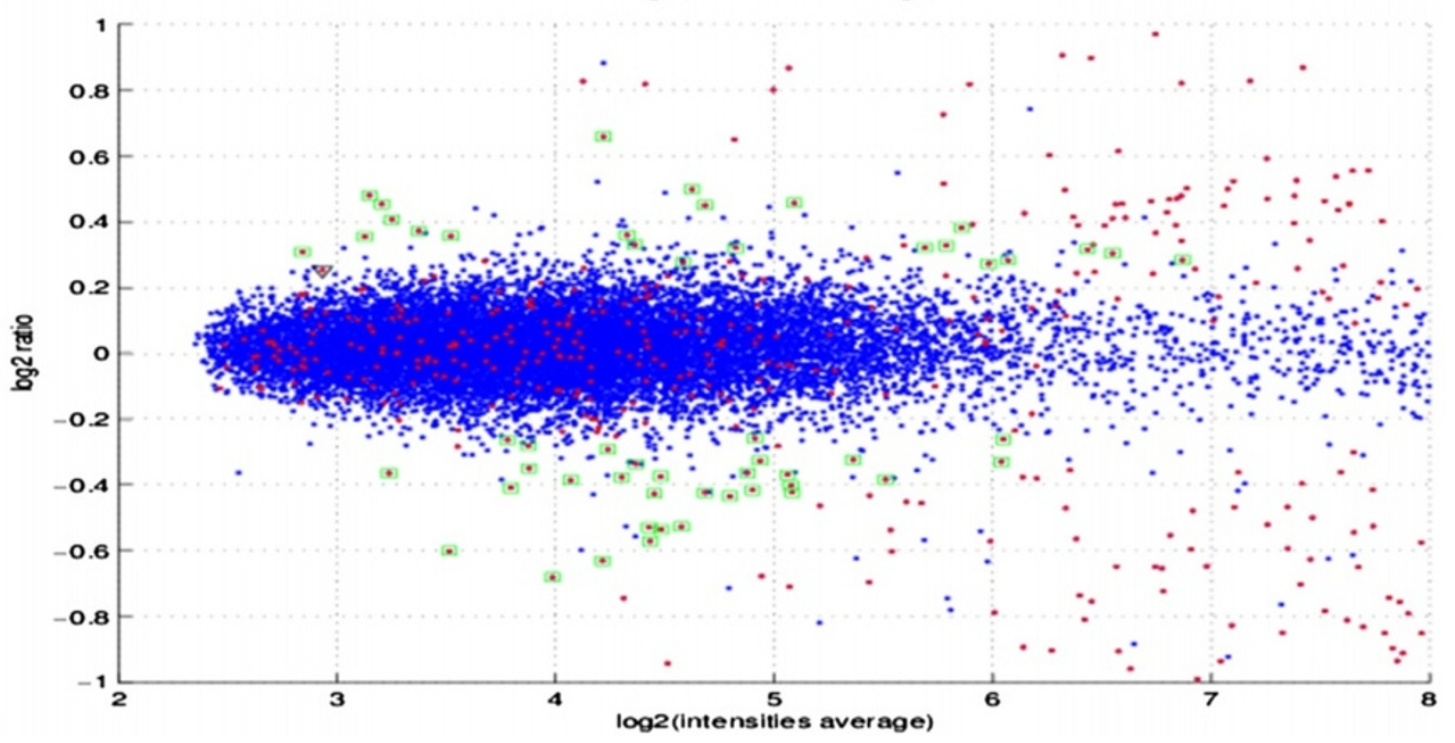

Figure 4 Comparison of the performance of methods with the Platinum Spike dataset. Platinum Spike dataset, Venn diagram of top 1940 genes by (A) the FCROS, RP, SAM and TREAT methods or (B) by the FCROS, FC, WAD and RP methods. Only genes that correspond to true DE genes are represented. M-A plot: (C) highly expressed genes where magenta diamonds are used for the 24 genes detected by the TREAT method (panel A), black triangles are used for the 15 genes detected by the SAM and TREAT methods (panel A), cyan circles are used for the 71 genes detected by the WAD method (panel B); and (D) weakly expressed genes where green squares are used for the 54 genes detected by the FCROS, FC and RP methods (panel B). Additional file 1: Figure S4 shows the full M-A plot of this dataset. 
Table 3 Comparative results for six microarray datasets (a)

\begin{tabular}{|c|c|c|c|c|c|}
\hline Dataset & Method & Thresholds & Selection & Runtime (s) & Error \\
\hline DLBCL & FCROS & $0.0228,0.9773$ & 424 & 4.79 & $4.55 \%$ \\
\hline$(n=7129)$ & $\mathrm{FC}$ & 1.444 & 425 & 0.13 & na \\
\hline \multirow[t]{5}{*}{$\left(m_{1}=58, m_{2}=19\right)$} & WAD & 1.1575 & 424 & 0.14 & na \\
\hline & $\mathrm{RP}$ & 0 & 425 & 656.36 & $0 \%$ \\
\hline & Ttest & 0.0001 & 428 & 1.48 & $0.17 \%$ \\
\hline & SAM & 0.00032 & 427 & 26.66 & $0.5 \%$ \\
\hline & TREAT & 0.0025 & 425 & 0.16 & $4.19 \%$ \\
\hline Prostate & FCROS & $0.0356,0.9644$ & 1009 & 19.58 & $7.12 \%$ \\
\hline$(n=12625)$ & $\mathrm{FC}$ & 1.27 & 1008 & 0.3 & na \\
\hline \multirow[t]{5}{*}{$\left(m_{1}=50, m_{2}=52\right)$} & WAD & 1.0805 & 1010 & 0.32 & na \\
\hline & $\mathrm{RP}$ & 0 & 1010 & 2491.54 & $0 \%$ \\
\hline & Ttest & 0.00068 & 1008 & 2.72 & $0.85 \%$ \\
\hline & SAM & 0.00041 & 1013 & 55.6 & $0.51 \%$ \\
\hline & TREAT & 0.0153 & 1010 & 0.33 & $19.12 \%$ \\
\hline Colon & FCROS & $0.0187,0.9802$ & 95 & 0.99 & $3.85 \%$ \\
\hline$(n=2000)$ & FC & 1.8 & 95 & 0.05 & na \\
\hline \multirow[t]{5}{*}{$\left(m_{1}=22, m_{2}=40\right)$} & WAD & 1.346 & 95 & 0.08 & na \\
\hline & $\mathrm{RP}$ & 0 & 96 & 168.78 & $0 \%$ \\
\hline & Ttest & 0.00015 & 97 & 0.5 & $0.3 \%$ \\
\hline & SAM & 0.00055 & 95 & 7.08 & $1.15 \%$ \\
\hline & TREAT & 0.00028 & 95 & 0.06 & $0.58 \%$ \\
\hline Leukemia & FCROS & $0.028,0.9717$ & 493 & 4.7 & $5.63 \%$ \\
\hline$(n=7129)$ & FC & 1.942 & 494 & 0.12 & na \\
\hline \multirow[t]{5}{*}{$\left(m_{1}=47, m_{2}=25\right)$} & WAD & 1.1668 & 494 & 0.17 & na \\
\hline & $\mathrm{RP}$ & 0 & 494 & 768.65 & $0 \%$ \\
\hline & Ttest & 0.00052 & 494 & 1.43 & $0.75 \%$ \\
\hline & SAM & 0.00051 & 494 & 28.71 & $0.74 \%$ \\
\hline & TREAT & 0.00153 & 494 & 0.13 & $2.2 \%$ \\
\hline Myeloma & FCROS & $0.01296,0.987$ & 450 & 34.77 & $2.59 \%$ \\
\hline$(n=12625)$ & $\mathrm{FC}$ & 1.5189 & 452 & 0.4 & na \\
\hline \multirow[t]{5}{*}{$\left(m_{1}=36, m_{2}=137\right)$} & WAD & 1.212 & 449 & 0.49 & na \\
\hline & $\mathrm{RP}$ & 0 & 451 & 4721.73 & $0 \%$ \\
\hline & Ttest & 0.0051 & 449 & 2.9 & $14.34 \%$ \\
\hline & SAM & 0.0055 & 450 & 94.84 & $15.43 \%$ \\
\hline & TREAT & 0.0192 & 451 & 0.54 & $53.75 \%$ \\
\hline ALL-1 & FCROS & $0.0355,0.9644$ & 1187 & 22.72 & $7.11 \%$ \\
\hline$(n=12625)$ & $\mathrm{FC}$ & 1.4176 & 1187 & 0.5 & na \\
\hline \multirow[t]{5}{*}{$\left(m_{1}=95, m_{2}=33\right)$} & WAD & 1.1165 & 1185 & 0.64 & na \\
\hline & $\mathrm{RP}$ & 0 & 1188 & 3108.43 & $0 \%$ \\
\hline & Ttest & 0.000057 & 1186 & 2.66 & $0.06 \%$ \\
\hline & SAM & 0.000145 & 1187 & 69.48 & $0.15 \%$ \\
\hline & TREAT & 0.00117 & 1184 & 0.26 & $1.25 \%$ \\
\hline
\end{tabular}


datasets (see above) to compare results obtained with the FCROS method and with the other six methods. To select the list of the most DE genes for each method, we used the results of the RP method, for which a PFP value of zero was associated to some genes. We determined the number of such genes and then set thresholds for the other methods to obtain a similar number of $\mathrm{DE}$ genes. We also recorded the runtime of each method. Results are summarized in Table 3. Errors for the Ttest, SAM and TREAT methods are obtained using $100 \alpha \frac{n}{n}$, where $n$ is the total number of genes, $\alpha$ the threshold used for the selection and $n_{2}$ the number of selected DE genes. For the FCROS method the error is given by $100\left(\alpha_{1}+1-\alpha_{2}\right)$, where $\alpha_{1}$ and $\alpha_{2}$ are the selection thresholds.

The results obtained show that the FC, WAD and TREAT methods have the smallest runtime followed by the Ttest and FCROS methods. The RP method has the largest runtime, which is more than 100 times higher than that of the FCROS method. The selection error (FDR) of each method is also shown in Table 3. Except for the Myeloma dataset, all errors are under 10\%. The error value for the RP method is the PFP. For the Prostate, the Myeloma and the ALL-1 datasets, we noted that the RP method detects some genes as down and up regulated at the same time. There are 19, 27 and 6 such genes for the Prostate, Myeloma and ALL-1 datasets, respectively. Most of these genes have a FC close to 1 . The bad detection performance of the RP method for these datasets probably comes from the number of permutations (100) used. An increase in this number will lead to an increase of the runtime which is already long. Thus, the RP method is suitable for a small number of samples but is not advisable for larger numbers of samples.

Table 4 shows the numbers of genes detected by all methods (common) and those uniquely detected by each method. The SAM and TREAT methods have the smallest number of genes not detected by any other method. We examined genes detected only by the Ttest, WAD or FC methods. The Ttest method detected some genes with a fold change close to 1 . The WAD method detected some highly expressed genes with a small FC but missed other genes with a high FC but low expression values. The FC method detected some genes for which only one sample has a large impact on the average values of all samples. Interestingly, for some datasets (e.g. Myeloma, Prostate, DLBCL), the FCROS method detected a relatively high number of probes not detected by other methods. The proportion of such probes varied highly between datasets, and was as high as $75 \%$ of the number of commonly detected probes (Myeloma dataset). This observation suggests that the FCROS method can significantly enrich the number of candidate DE genes.

We conducted a close inspection of the Colon and the Prostate datasets and used the interactive Venn Diagram plotter software [40] to search for specific and common DE genes detected by the FCROS, RP, SAM and TREAT methods. As expected, the FCROS method shared more genes with the RP method than with the SAM or TREAT methods. Figure 5A shows that 58 genes were detected by these four methods for the Colon dataset. The FCROS method also shared 16 genes with the SAM and the TREAT methods, which were not detected by the RP method. In contrast, no gene was detected jointly by the RP, SAM or TREAT methods which was not detected by the FCROS method. For the Prostate dataset (Figure 5B), 519 genes were detected by all four methods. Many genes were detected only by the FCROS (105) or shared by the FCROS, SAM and TREAT methods (161), and not detected by the RP method. Again, only few genes (12) were detected by the RP and the SAM or TREAT methods that were not detected by FCROS. We further used the Prostate dataset to compare the 4 FC based methods (Figure 5C). We considered that genes that were detected by 2 or more methods are good candidates for true $\mathrm{DE}$ genes. There were 1095 such genes, of which $56 \%$ were commonly identified by all methods. No method clearly outperformed the other, as each of them failed to detect $\approx$ $10 \%$ of genes that were detected by at least 2 of the other methods. Surpringly, the simple FC method detected the fewest number of genes that were not detected by other methods.

Effect of sample number A literature survey performed in [41] shows that many biological microarray studies use

Table 4 Comparative results for six microarray datasets (b)

\begin{tabular}{lllllllll}
\hline Dataset & Common & FCROS & FC & WAD & RP & Ttest & SAM & TREAT \\
\hline DLBCL & 149 & 22 & 79 & 54 & 31 & 99 & 5 & 0 \\
Prostate & 308 & 47 & 58 & 81 & 90 & 183 & 1 & 1 \\
Colon & 39 & 1 & 9 & 10 & 9 & 10 & 0 & 4 \\
Leukemia & 191 & 14 & 89 & 102 & 43 & 44 & 11 & 0 \\
Myeloma & 70 & 53 & 162 & 47 & 124 & 108 & 5 & 1 \\
ALL-1 & 640 & 26 & 86 & 131 & 55 & 131 & 10 & 0
\end{tabular}

Common and specific number of genes detected by the seven methods in the six datasets. 


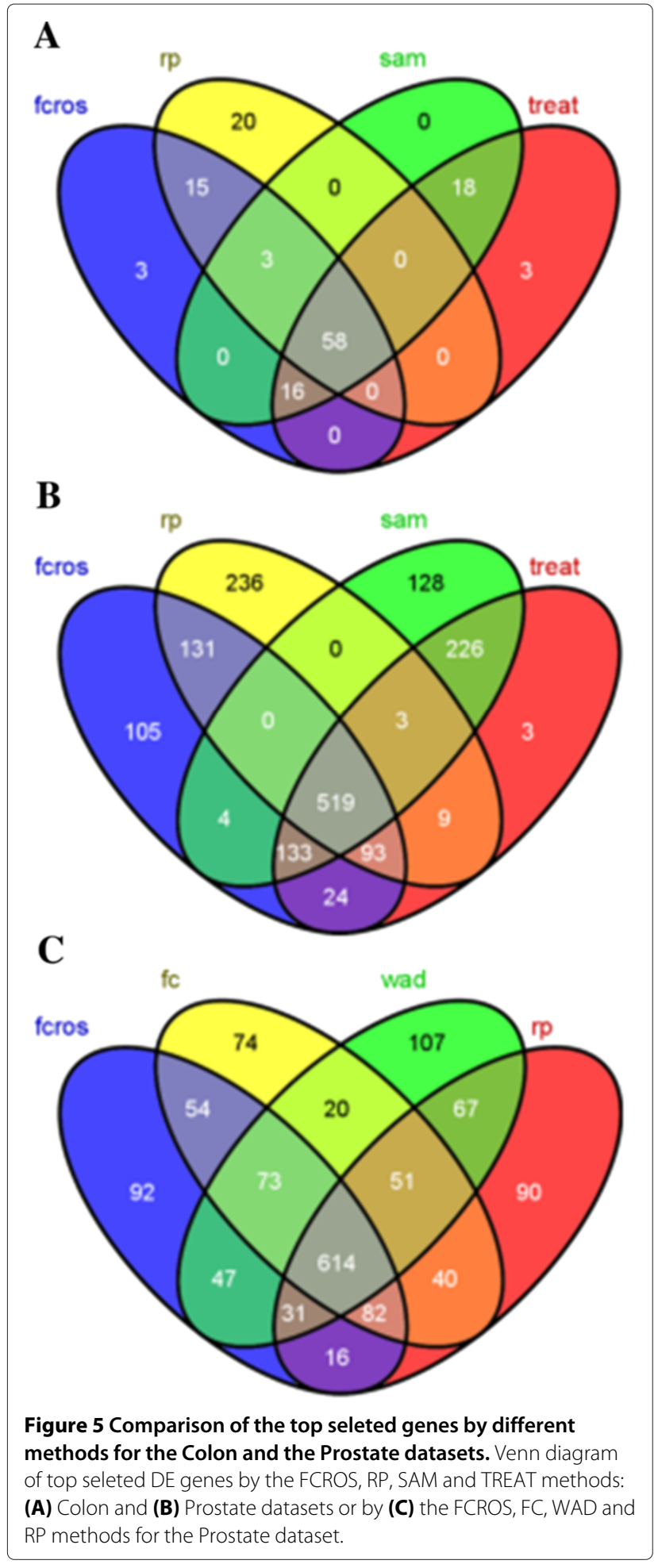

very small number of replicates (e.g. 3 to 5). To evaluate the consequence of such choices on the detection power, we used the Colon dataset, and conducted analyses with varying numbers of control and test samples selected from the original dataset. In a first analysis and for a given $\left(m_{1}, m_{2}\right)$ pair, we proceeded as follows: a) random selection of $m_{1}$ control and $m_{2}$ test samples from their true sample groups, b) run the seven methods to obtain results for each, c) select the 100 top DE probes and assign 1 to them and 0 to all other probes. These three steps were repeated 100 times and the total occurences of 1 for each probe was calculated as its score. To set a threshold for the score, we performed a second analysis where control and test samples were chosen without regard for the biological sample groups to which they belong. High scores, in interval $\left[S_{t h r}, 100\right]$, are expected for the DE genes in the first analysis. Small scores, in interval $\left[0, S_{t h r}\right]$, should be associated to all genes in the second analysis. $S_{t h r}$ is the score threshold which varies with the method used. We sorted genes using their scores in each analysis.

Figure 6 shows the results from these analyses. For each setting for control and test samples, we ordered genes according their scores when control and test samples are selected from their true sample groups (green line) or without regard for that true group (red line). Based on these results, we used respectively the score thresholds $S_{t h r}$ of 40,70,60,60,17, 20 and 18 for the FCROS, FC, WAD, RP, Ttest, SAM and TREAT methods. These thresholds allow to obtain the results illustrated in Figure 7, which shows that rank based methods (FCROS, FC, WAD and RP) select fewer genes than the Ttest, SAM and TREAT methods. The FCROS method detects more genes than the other FC based methods. Results depicted in Figure 6 show that the Ttest, SAM and TREAT methods associated non zero score values to more genes, as revealed by the departure from zero in the $\mathrm{x}$-axis of the score plots. The RP method identifies genes with a high score for completely random control and test samples, indicating its propensity to detect false positives. A similar trend, less pronounced, is also observed for the FC and WAD methods. The WAD method, however, assigned high scores to more genes than the other methods (especially for the $3 \times 3$ and $5 \times 5$ cases), confirming the high degree of reproducibility of this method [12].

We recorded the values observed for parameters $\delta$ for all runs of FCROS using different settings for $m_{1}$ and $m_{2}$. We plotted in Additional file 1: Figure S5 the results obtained for different settings for the number of control and test samples. As for the synthetic datasets, we observed higher values for $\delta$, greater than 0.9 , when control and test samples are not randomized. However, these values vary more than those obtained with the synthetic datasets. When control and test samples are randomized, values for $\delta$ decrease towards 0.6 when the number of samples used increases.

Analysis of reproducibility We further used the Colon dataset to assess the reproducibility of DE genes identification in a complex noisy dataset. We conducted 100 runs, in which we used half of the dataset, i.e. 11 control and 

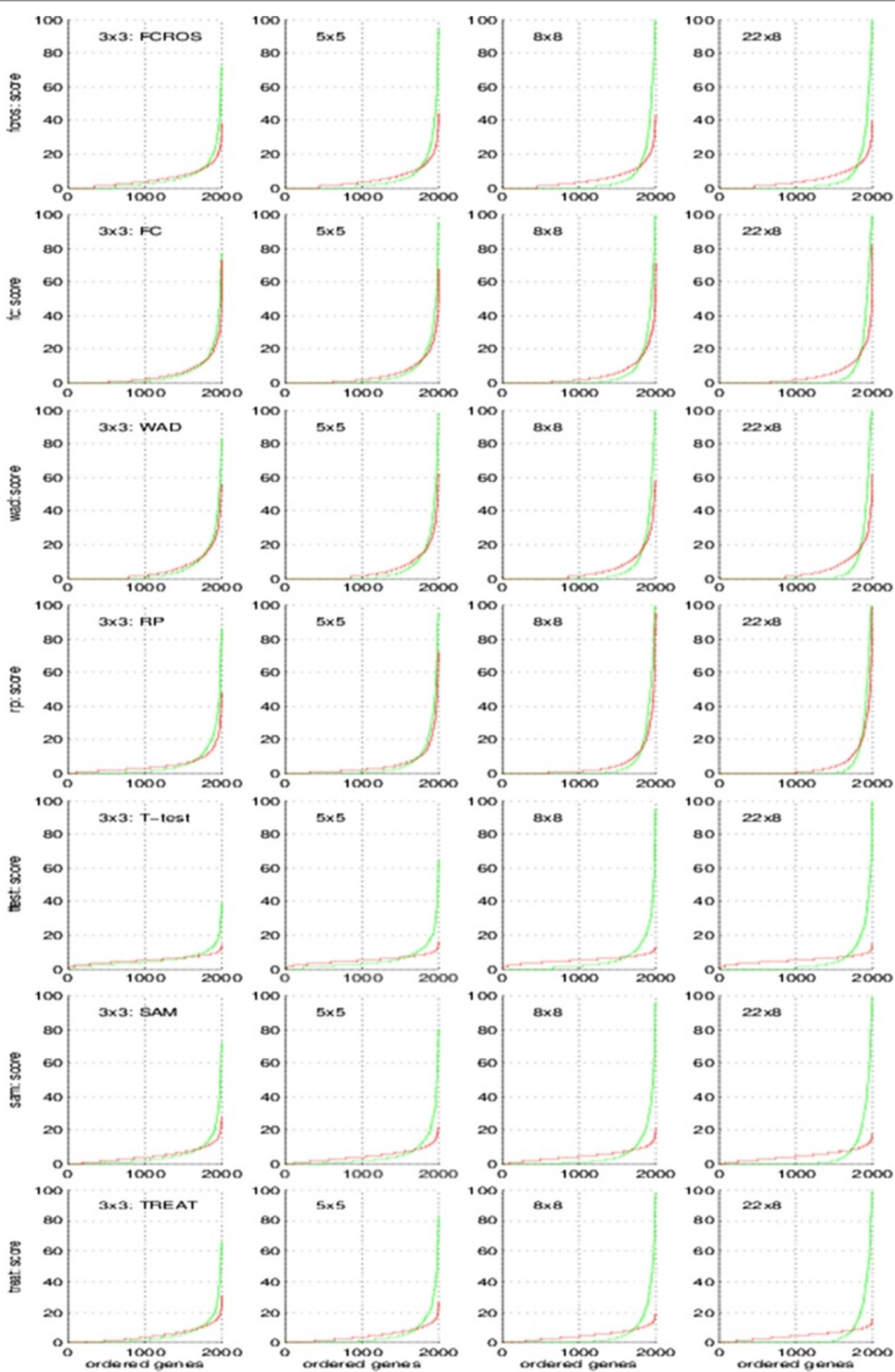

Figure 6 Impact of sample group size on the performance of the methods. Colon dataset, plot of scores obtained using the seven methods. Genes in the abscissa are ordered according to their score. The green line is used for the random selection of the control and the test samples from their true sample groups. The red line is used if no distinction was done between true sample groups during the selection of the control and the test samples. 


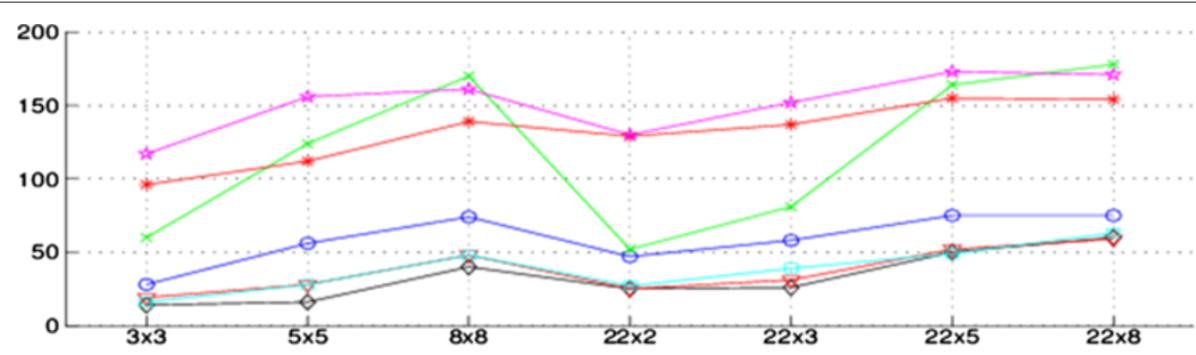

Figure 7 Number of DE genes selected for the Colon dataset by the seven methods. Colon dataset, number of DE genes selected using the FCROS (blue circle line), FC (black diamond line), WAD (red triangle line), RP (cyan square line), Student t-test (green $x$-mark line), SAM (red star line) and TREAT (magenta pentagram line) methods.

20 test samples that were randomly selected. The top 100 genes identified as DE in each run were assigned a score of 1 while all other genes were assigned a score of 0 . The overall score for each gene was calculated as the sum of its scores. We evaluated the reproducibility of each method by counting the number of genes with perfect (100) or good $(\geq 90)$ global scores (Table 5). As expected, the FC based methods were better than the t-test based methods in reproducibly identifying DE genes. Among these methods, FCROS and WAD were more reproducible than FC and RP.

\section{Conclusion}

We have described here a new FC-based method and shown that it is powerful to detect $\mathrm{DE}$ genes in noisy datasets. Importantly, the FCROS method assigns a statistic to DE genes, which can be used as a selection criterion. FCROS appears to be more specific and much faster than RP, and as sensitive and reproducible as WAD. The FCROS method has two possible applications, when used in combination with other methods: 1) identification of a core set of high confidence DE genes detected by all methods, 2) identification of additional potentially DE genes not detected by other methods. This last possibility may be especially relevant when studying samples with a high degree of intrinsic biological variability (like tumor samples). Our results indeed show that the FCROS method can detect many DE genes in tumor datasets, which escape identification with other methods (Figure 5). In studies of rare diseases, the number of patient samples can be very low while the number of control samples from healthy people is high. The results from Figures 3 and 7 show that the FCROS method performs well in such

Table 5 Comparative results for the Colon dataset

\begin{tabular}{llllllll}
\hline \multicolumn{7}{c}{ Number of genes } \\
\hline Global score & FCROS & FC & WAD & RP & Ttest & SAM & TREAT \\
\hline 100 & 14 & 12 & 13 & 13 & 5 & 5 & 6 \\
$\geq 90$ & 34 & 26 & 37 & 28 & 13 & 17 & 19 \\
\hline
\end{tabular}

Colon dataset: scores obtained using the seven methods. situations. The FCROS method has also other advantages. (1) It does not require prefiltering to improve the statistic associated with each gene. In contrast, prefiltering is important for other methods, as it decreases the computational load and the FDR. (2) In contrast to the SAM and the RP methods, for which the results can vary from one run to another, the FCROS method is deterministic. (3) The FCROS method can be easily adapted for data originaly from different experiments for which batch related biases can often not be completely corrected by normalization methods. FCROS does not require interbatch normalization. For instance, if the data are from two experimental batches, we can use $k=k_{1}+k_{2}$ comparisons where $k_{1}$ and $k_{2}$ are the numbers of pairwise comparisons from the first and the second batch, respectively.

We provide an $\mathrm{R}$ package which is deposed on the Comprehensible R Archive Network (CRAN) server for download, see http://www.r-project.org. The function fcros2() allows to deal with datasets from two batches. Usage of the package $f$ cros is available in the help function.

\section{Additional file}

Additional file 1: Supplementary figures.

Competing interests

The authors declare that they have no competing interests.

\section{Authors' contributions}

DD drafted the paper and performed the analyses. Both authors developed the method and contributed to the manuscript. Both authors read and approved the final manuscript.

\section{Acknowledgements}

We thank S. Chan for critical reading and editing the manuscript. This work was supported by funds from INSERM, CNRS and Université de Strasbourg.

\section{Author details \\ 'Institut de Génétique et de Biologie Moléculaire et Cellulaire (IGBMC), INSERM U964, CNRS UMR 7104, Université de Strasbourg, 67404 Illkirch, France. ${ }^{2}$ IGBMC Microarray and Sequencing Platform, 67404 IIIkirch, France. ${ }^{3}$ Faculté de Medécine, Université de Strasbourg, Strasbourg, France.}

Received: 3 July 2013 Accepted: 27 December 2013

Published: 15 January 2014 
References

1. Schena M, Shalon D, Davis RW, Brown PO: Quantitative monitoring of gene expression patterns with a complementary DNA microarray. Science 1995, 270(5235):467-470.

2. Lockhart DJ, Dong H, Byrne MC, Follettie MT, Gallo MV, Chee MS Mittmann M, Wang C, Kobayashi M, Norton H, Brown EL: Expression monitoring by hybridization to high-density oligonucleotide arrays. Nat Biotechnol 1996, 14:1675-1680.

3. Peart MJ, Smyth GK, van Laar RK, Bowtell DD, Richon VM, Marks PA, Holloway AJ, Johnstone RW: Identification and functional significance of genes regulated structurally different histone deacetylase inhibitors. Proc Nat Acad Sci USA 2005, 102(10):3697-3702

4. Patterson TA, Lobenhofer EK, Fulmer-Smentek SB, Collins PJ, Chu TM, Bao W, Fang H, Kawasaki ES, Hager J, Tikhonova IR, Walker SJ, Zhang L, Hurban P, de Longueville F, Fuscoe JC, Tong W, Shi L, Wolfinger RD: Perfomance comparison of one-color and two-color platforms within the Microarray Quality Ccontrol (MAQC) projet. Nat Biotechnol 2006, 24(9):1140-1150.

5. Golub TR, Slonim DK, Tamayo P, Huard C, Gaasenbeek M, Mesirov JP, Coller H, Loh ML, Downing JR, Caligiuri MA, Bloomfield CD, Lander ES: Molecular classification of cancer : class discovery and class prediction by gene epression monitoring. Science 1999, 286:531-537.

6. Tusher VG, Tibshirani R, Chu G: Significance analysis of microarrays applied to the ionizing radiation response. Proc Nat Acd Sci USA 2001, 98(9):5116-5121.

7. Baldi $P$, Tong AD: A bayesian framework for the analysis of microarray expression data: regularized t-test and statistical inference of gene changes. Bioinformatics 2001, 17(6):509-519.

8. Ambroise C, McLachlan GJ: Slection bias in gene extraction on the basis of microarray gene-expression data. Proc Natl Acad Sci USA 2002, 99(10):6562-6566.

9. Smyth $\mathrm{G}$ : Linear models and empirical bayes methods for assessing differential expression in microarray experiments. Stat App/ Genet Mol Biol 2004, 3(1):article 3.

10. Pan W: A comparative review of statistical methods for discovery differentially expressed genes in replicated microarray experiments. Bioinformatics 2002, 18(4):546-554.

11. Jeffery IB, Higgins DG, Culhane AC: Comparison and evaluation of methods for generating differentially expressed gene lists from microarray data. BMC Bioinformatics 2006, 7(359):1471-2105.

12. Kadota $K$, Nakai $Y$, Shimizu $K$ : A weighted average difference method for detecting differentiallly expressed genes from microarray data. BMC Algorithms Mol Biol 2008, 3(8):1748-7188.

13. Kadota K, Shimizu K: Evaluating methods for ranking differentially expressed genes applied to microArray quality control data. BMC Bioinformatics 2011, 12(227):1471-2105.

14. Guo L, Lobenhofer EK, Wang C, Shippy R, Harris SC, Zhang L, Mei N, Chen T, Herman D, Goodsaid FM, Hurban P, Phillips KL, Xu J, Deng X, Sun YA, Tong W, Dragan YP, Shi L: Rat toxicogenomic study reveals analytical consistency across microarray platforms. Nat Biotechnol 2006, 24(9):1162-1169.

15. Shi L, Tong W, Fang H, Scherf U, Han J, Puri RK, Frueh FW, Goodsaid FM, Guo L, Su Z, Han T, Fuscoe JC, Xu ZA, Patterson TA, Hong H, Xie Q, Perkins RG, Chen JJ, Casciano DA: Cross-platform comparability of microarray technology: Intra-platform consistency and appropriate data analysis procedure are esential. BMC Bioinformatics 2005, 6(Suppl 2):1471-2105

16. Chen JJ, Hsueh HM, Delongchamp RR, Lin CJ, Tsai CA: Reproducibility of microarray data: a further analysis of microarray quality control (MAQC) data. BMC Bioinformatics 2007, 8(412):1471-2105.

17. Farztdinov V, McDyer F: Distributional fold change test - a statistical approach for detecting differential expression in microarray experiments. Algorithms Mol Biol 2012, 7(29):1-16.

18. McCarthy DJ, Smyth GK: Testing significance relative to a fold-change threshold is a TREAT. Bioinformatics 2009, 25(6):765-771.

19. Yanofsky CM, Bickel DR: Validation of differential gene expression algorithms: application comparing fold-change estimation to hypothesis testing. BMC Bioinformatics 2010, 11(63):1471-2105.

20. Xiao Y, Hsiao TH, Suresh U, Chen HIH, Wu X, Wolf SE, Chen Y: A novel significance score for gene selection and ranking. Bioinformatics 2012. doi:10.1093/bioinformatics/btr671.
21. Qi Y, Sun H, Sun Q, Pan L: Ranking analysis for identifying differentially expresed genes. Genomics 2011, 97:326-329.

22. Breitling R, Armengaud P, Amtmann A, Herzyk P: Rank products: a simple, yet powerful, new method to detect differentially expressed genes in replicated microarray experiments. FEBS Lett 2004, 573:83-92

23. Eisinga $R$, Breitling $R$, Heskes $T$ : The exact probability distribution of the rank product statistics for replicated experiments. FEBS Lett 2013, 587:677-682

24. Feller W: An Inroduction to Probability Theory and Its Applications, Volume 2, 2nd Edition. New York, USA: John Wiley \& Sons; 1970.

25. R Core Team: The R-roject for Statistical Computing. [http://www.rproject.org/] (accessed on 17 April 2013)

26. Tibshirani R, Chu G, Narasimhan B, Li J: samr: SAM: Significance analysis of microarrays. [http://cran.r-project.org/web/packages/samr/index html] (accessed on 17 April 2013).

27. Hong F, Wittner B, Breitling R, Smith C, Battke F: Bioconductor RankProd package. [http://cran.r-project.org/web/packages/samr/index.html] (accessed on 17 April 2013)

28. Carey V, Redestig H: ROC: utilities for ROC, with microarray focus. [http://www.bioconductor.org] R package version 1.36 .0 (accessed on 16 september 2013)

29. Dembélé D: A flexible microarray data simulation model. Microarrays 2013, 2(2):115-130.

30. Dembélé $D$ : R package madsim: A flexible microarray data simulation model. [http://cran.r-project.org/web/packages/madsim/] (accessed on 17 april 2013).

31. Dudoit S, Yang YH, Callow MJ, Speed TP. Statistical methods for identifying differentially expressed genes in replicated cDNA microarray experiments. Stat $\sin 2002,12: 111-139$.

32. Zhu Q, Miecznikowski JC, Halfon MS: Preferred analysis methods for affymetrix geneChips. II. An expanded, balanced, wholly-defined spike-in dataset. BMC Bioinformatics 2010, 11(285):1471-2105.

33. Jeffery IB: Post-procesed microarray datasets. [http://www.bioinf.ucd. ie/people/ian/] (accessed on 17 april 2013)

34. Shipp MA, Ross KN, Tamayo P, Weng AP, Kutok JL, Aguiar RC, Gaasenbeek M, Angelo M, Reich M, Pinkus GS, Ray TS, Koval MA, Last KW, Norton A, Lister TA, Mesirov J, Neuberg DS, Lander ES, Aster JC, Golub TR: Diffuse large B-cell lymphoma outcome prediction by geneexpression profiling and supervised machine learning. Nat Med 2002, 8:68-74

35. Singh D, Febbo PG, Ross K, Jackson DG, Manola J, Ladd C, Tamayo P, Renshaw AA, D'Amico AV, Richie JP, Lander ES, Loda M, Kantoff PW, Golub TR, Sellers WR: Gene expression correlates of clinical prostate cancer behavior. Cancer Cell 2002, 1(2):203-209.

36. Alon U, Barkai N, Notterman DA, Gish K, Ybarra S, Mack D, Levine AJ: Broad patterns of gene expression revealed by clustering analysis of tumor and normal colon tissues probed by oligonucleotide arrays. Proc Natl Acad Sci USA 1999, 96(10):6745-6750.

37. Tian E, Zhan F, Walker R, Rasmussen E, Ma Y, Jr Barlogie B: JDS: The role of the Wnt-Signaling antagonist DKK1 in the development of osteolytic lesions in multiple myeloma. N Eng J Med 2003, 349(26):2483-2494

38. Chiaretti S, Li X, Gentleman R, Vitale A, Vignetti M, Mandelli F, Ritz J, Foa R: Gene expression profile of adult T-cell acute lymphocytic leukemia identifies distinct subsets of patients with different response to therapy and survival. Blood 2004, 103(7):2771-2778.

39. Irizarry RA, Hobbs B, Collin F, Beazer-Barclay YD, Antonellis KJ, Scherf U, Speed TP: Exploration, normalization and summaries of high-density oligonucleotide array probe level data. Biostatistics 2003, 4(2):249-264

40. Oliveros J: VENNY. An interactive tool for comparing lists with Venn Diagrams. [http://bioinfogp.cnb.csic.es/tools/venny/] (accessed on 24 april 2013).

41. Breitling R, Herzyk P: Rank-based methods as a non-parametric alternative of the t-statistic for the analysis of biological microarray data. J Bioinform Comput Biol 2005, 3(5):1171-1189.

doi:10.1186/1471-2105-15-14

Cite this article as: Dembélé and Kastner: Fold change rank ordering statistics: a new method for detecting differentially expressed genes. BMC Bioinformatics 2014 15:14 